ORIGINAL

\title{
Ocorrência de lesões podais e graus de claudicação em vacas lactantes da raça Jersey mantidas em sistema free-stall e semiconfinamento
}

\author{
Occurrence of foot injuries and degrees of claudication in lactating \\ Jersey cows kept in a free-stall and semi-confinement system
}

Francisco Armando de Azevedo Souza ${ }^{1}$, Juliana das Chagas Goulart Correio ${ }^{1}$, Thais Helena Constantino Patelli ${ }^{1}$, Emília de Paiva Porto ${ }^{1}$, Vitor Bruno Bianconi Rosa ${ }^{1}$, Bruna Fonseca Matias Correio ${ }^{2 *}$, José Victor Pronievicz Barreto $^{2}$, Simone Fernanda Nedel Pértile ${ }^{2}$, Gustavo Rodrigues Queiroz ${ }^{2}$, Luiz Fernando Coelho da Cunha Filho ${ }^{2}$

${ }^{1}$ Universidade Estadual do Norte do Paraná (UENP), Bandeirantes, PR, Brasil

2 Universidade Pitágoras Unopar, Arapongas, PR, Brasil

\section{Resumo}

Dependendo da extensão, as lesões dificultam o movimento do animal afetado, limitando seu acesso ao alimento e à água e resultando na redução de seu bemestar, produção e rentabilidade. Este trabalho teve como objetivo avaliar se os sistemas de confinamento freestall e semiconfinamento influenciam na ocorrência de afecções podais e no grau de claudicação de vacas lactantes da raça Jersey. Entre os animais examinados, todos apresentaram algum tipo de lesão, sendo visualizadas lesões múltiplas na maioria deles. Contudo, $86,33 \%$ dos animais no sistema free-stall e $84,78 \%$ no semiconfinamento não apresentaram claudicação. Os resultados demonstram que a média das lesões por vaca foi de 19,8 no sistema free-stall e de 15,67 no sistema de semiconfinamento. Em ambos os sistemas, a maior distribuição das lesões se encontrava no membro pélvico. Conclui-se que os sistemas de produção nas duas propriedades não diferiram entre si na ocorrência de afecções podais em vacas lactantes da raça Jersey. Além disto, a existência de lesões podais não foi correlacionada diretamente com claudicação.

Palavras-chave: Afecções podais. Escore de claudicação. Sistema intensivo. Sistema semi-intensivo. Vacas leiteiras.

\begin{abstract}
Claudication dificults the movement of the affected animal by limiting its access to food and water, resulting in reduced welfare, production and profitability. The objective of this study was to evaluate whether the free-stall and semiconfinement systems influence the occurrence of foot affections and degrees of claudication of lactating Jersey
\end{abstract}


cows. Among the animals examined, all presented some type of lesion, with multiple lesions being seen in most animals. However, $86.33 \%$ animals in the free-stall system and $84.78 \%$ in the semi-confinement did not present lameness. The results show that the mean number oflesions per cow was 19.8 lesions in the free-stall system, and 15.67 in the semi-confinement sysrtem. In both systems, the largest distribution of lesions was found in the pelvic limb. It was concluded that the free-stall and semi-confinement production system do not differ in the occurrence of foot affections in lactating Jersey cows. In addition, the existence of foot lesions is not directly correlated with lameness.

Keywords: Foot conditions. Claudication score. Intensive system. Semi-intensive system. Dairy cows.

\section{Introdução}

As lesões podais em bovinos leiteiros são problemas considerados de importância, devido à intensificação dos sistemas de exploração que comprometem a produtividade do rebanho, bem como representam uma ameaça ao bem-estar animal (Cerqueira et al., 2011; Martins e Pieruzzi, 2011; Roman et al., 2014). Os principais prejuízos devido à presença das lesões podais são: queda na produção de leite, redução da fertilidade, descarte precoce dos animais e altos custos com tratamentos. Além disso, podem influenciar na incidência de outras doenças como mastite e metrite (Nicoletti et al., 2001; Nicoletti, 2004; Silva et al., 2004; Alves, et al. 2007; Roman et al., 2014; Rutter, 2015; Souza et al., 2015), assim como no sofrimento e bem-estar dos animais.

As etiologias das enfermidades podais são múltiplas, porém há uma relação direta da incidência com o tipo de sistema de produção envolvido, com a produção leiteira e com o número de partos (Silva, et al., 2010). A maior concentração de animais por área resulta em grandes volumes de fezes e urina, o que acarreta em maior umidade, menor higiene no ambiente e maior contaminação por agentes infecciosos como Fusobacterium necrophorum, Dichelobacter nodosus e Treponema sp. (Greenough, 2007). Associado ao tipo de piso e às camas inadequadas, o manejo fica mais difícil e faz com que as vacas fiquem mais tempo em posição quadrupedal, sobrecarregando os membros e predispondo a lesões podais (Nicoletti, 2004; Pozzatti et al., 2010; Plautz, 2013; Costa et al., 2014). Em sistemas de produção semi-intensivos, onde os animais ficam parte do tempo a pasto, ocorre um desgaste mais uniforme do casco. Deve-se observar, contudo, fatores de risco como o acúmulo de água e barro em áreas com declividade ou próximos a cocheiras de água e sal, além das estradas que conduzem os animais até o confinamento, pois a presença de buracos e/ou pedras predispõe a traumas (Borges e Garcia, 2002; Borkert, 2015).

Segundo Nicoletti (2004), claudicações são causadas em $90 \%$ dos casos por lesões podais. Um rebanho que possui de $7 \%$ a $10 \%$ de claudicação é considerado aceitável, porém estes índices podem ser superados e variam de acordo com a região. $\mathrm{O}$ autor ainda observou que a claudicação é considerada a terceira causa de descartes de animais, superada apenas pelos problemas reprodutivos e pela mastite. Por outro lado, Silva et al. (2008), ao trabalharem com vacas da raça Holandesa, verificaram que houve um descarte de $18,5 \%$ dos animais por problemas no sistema locomotor, ocupando o segundo lugar em causas de descarte de animais.

Este trabalho teve como objetivo avaliar se os sistemas de confinamento free-stall e semiconfinamento influenciam na ocorrência de afecções podais e no grau de claudicação de vacas lactantes da raça Jersey.

\section{Materiais e métodos}

O estudo foi realizado em rebanhos da raça Jersey de duas propriedades localizadas na mesorregião centro-oriental do estado do Paraná.

A primeira propriedade, situada no município de Ponta Grossa (25 05’ 42" S, 50 09' 43" W), continha 135 animais em lactação, em sistema de confinamento free-stall, com piso de concreto, cama com colchão de borracha, coberto com maravalha, e acesso a piquete com Cynodon sp. A ordenha era realizada mecanicamente, duas vezes ao dia, com produção média de $26 \mathrm{~kg} / \mathrm{vaca} /$ dia. A alimentação era constituída por volumoso de silagem de milho, 
pré-secado de azevém, ração peletizada, farelo de soja e farelo de milho. Foram avaliados 22 animais, sendo 11 vacas primíparas, com idade média de 31 meses, e 11 vacas multíparas, com idade entre 4 e 10 anos. Todos os animais se encontravam em lactação e foram escolhidos aleatoriamente para a realização do experimento.

A segunda propriedade, situada no município de Ventania (24 $\left.14^{\prime} 45^{\prime \prime} \mathrm{S}, 50^{\circ} 14^{\prime} 34^{\prime \prime} \mathrm{W}\right)$, possuía 125 animais em lactação, em sistema de semiconfinamento, sendo confinados durante 8 horas por dia e o restante do tempo a pasto de gramínea Cynodon sp. A ordenha era realizada mecanicamente duas vezes ao dia, com produção média de $16 \mathrm{~kg} / \mathrm{vaca} /$ dia. Além do pasto de Cynodon sp., também eram fornecidos aveia, farelo de soja e farelo de milho. Nesta fazenda foram examinadas 46 vacas em lactação, sendo 23 primíparas, com idade média de 30 meses, e 23 multíparas, com idade entre 4 e 10 anos, escolhidas aleatoriamente.

Ambas as propriedades utilizavam pedilúvio todos os dias após a ordenha, com sulfato de cobre na concentração de $5 \%$, sendo a troca do produto realizada a cada cinco dias. 0 casqueamento era realizado somente quando detectadas alterações visíveis no casco ou claudicação.

Após a seleção, cada vaca foi colocada em um tronco de contenção. Durante o trajeto, observou-se a locomoção a fim de avaliar o grau de claudicação e classificá-la de acordo com o seguinte escore: (1) normal - ausência de claudicação; (2) claudicação leve; (3) claudicação moderada; (4) claudicação grave; (5) impotência funcional - quando o animal é incapaz de apoiar o membro (Greenough e Weaver, 1997).

Após a limpeza dos dígitos com água e escova, procedeu-se à avaliação clínica e identificação das lesões. Os dígitos foram avaliados separadamente ( $\mathrm{n}=544$ ) e os dados foram anotados em ficha individual específica de lesões podais (Anexos $1 \mathrm{e}$ 2), onde pode-se indicar o local da lesão e o membro acometido. Conforme Nicoletti et al. (2001), as lesões foram classificadas em hemorragia de sola, úlcera de sola, doença da linha branca, erosão de talão, sola dupla, abscesso sub-solear, flegmão interdigital, tiloma ou hiperplasia interdigital, dermatite interdigital, dermatite digital, artrite séptica, rachadura vertical, rachadura horizontal, pinça longa/talão baixo e sola plana.

As análises estatísticas foram realizadas no procedimento GLIMMIX do programa estatístico SAS (University Edition, SAS Institute Inc., Cary, NC). As análises de variância foram realizadas utilizando a metodologia de modelos lineares generalizados, considerando a distribuição Binomial para a característica média de lesões por vaca e a distribuição Bernoulli para as outras características estudadas. 0 modelo utilizado foi composto pelos efeitos fixos de fazenda, número de partos (primíparas ou multíparas), dígito e interação entre fazenda e número de partos. 0 teste de comparação de médias utilizado foi Tukey. Para todos os testes foi considerado um nível de significância de $5 \%$.

\section{Resultados e discussão}

Entre os animais examinados, todos apresentaram algum tipo de lesão, sendo visualizadas lesões múltiplas na grande maioria. Contudo, $86,33 \%$ dos animais no sistema free-stall e $84,78 \%$ no semiconfinamento não apresentaram claudicação (G1). Esses dados diferem um pouco dos apresentados por Nicoletti et al. (2001) em estudo realizado com vacas Holandesas, onde 77,7\% dos animais com lesões podais não apresentaram claudicação.

Os resultados demonstraram que os sistemas de manejo free-stall e de semiconfinamento não diferiram quanto ao número de lesões podais e escore de claudicação (Tabela 1). Nicoletti et al. (2001), estudando os sistemas de confinamento free-stall e tie-stall, concluíram também que não houve diferença do número de lesões e grau de claudicação entre estes sistemas. Entretanto, no presente estudo, ao se comparar o sistema de semiconfinamento com o free-stall, obtevese um número maior de lesões podais do que em relação ao estudo de Nicoletti et. al (2001). Isto provavelmente ocorreu devido à maior concentração de animais por área, gerando acúmulo de dejetos (Greenough, 2007; Tomasella et al., 2014). 
Tabela 1 - Número de lesões observadas, média de lesões por vaca e graus de claudicação (G) nos dois sistemas de produção

\begin{tabular}{lcccccccc}
\hline Sistema & Número de vacas & $\mathbf{N}^{0}$ de lesões & Média de lesões/vaca* & G1 (\%) & G2 (\%) & G3 (\%) & G4 (\%) & G5 (\%) \\
\hline Free-stall & 22 & 436 & 19,8 & 86,36 & 4,54 & 9,10 & 0 & 0 \\
Semiconfinamento & 46 & 721 & 15,67 & 84,78 & 8,69 & 6,53 & 0 & 0 \\
Total & 68 & 1157 & 17,74 & - & - & - & - & - \\
\hline
\end{tabular}

Nota: $\mathrm{G1}$ = normal - ausência de claudicação; G2 = claudicação leve; $\mathrm{G} 3$ = claudicação moderada; $\mathrm{G4}$ = claudicação grave; G5 = impotência funcional - quando 0 animal é incapaz de apoiar o membro (Greenough e Weaver, 1997).

Os resultados demonstram que a média das lesões por vaca foi de 19,8 lesões no sistema freestall e de 15,67 no sistema de semiconfinamento, e que estas não diferiram estatisticamente $(\mathrm{p}<0,05)$.

Com relação à distribuição de lesões nos dígitos, das 436 lesões encontradas nos animais em sistema free-stall, 58,9\% estavam localizadas nos membros pélvicos, com $55,25 \%$ de acometimento dos dígitos laterais. Estes resultados são semelhantes aos encontrados por Silva et al. (2010), que observaram uma média de $57 \%$ das lesões localizadas nos membros pélvicos, porém são menores do que os encontrados por Tomasella et al. (2014) (87,5\%). 0 maior acometimento dos membros pélvicos se deve, provavelmente, ao maior contato com fezes, urina e excesso de umidade. As lesões em membros anteriores corresponderam a 41,05\%, e o acometimento entre os dígitos mediais e laterais, foi semelhante neste estudo. Outro fator a ser salientado é o deslocamento do centro de gravidade para os membros pélvicos, devido ao peso do úbere, o que pode contribuir para a maior ocorrência de lesões podais em membros pélvicos.

No sistema de semiconfinamento foram observadas 721 lesões, das quais 379 (52,57\%) encontravam-se nos membros pélvicos, com acometimento dos dígitos laterais de 50,66\%. Estes resultados diferem dos descritos por Romani et al. (2004), que observaram $75,3 \%$ de lesões em membros pélvicos em vacas mestiças. As lesões em membros torácicos corresponderam a $47,43 \%$ e o acometimento entre os dígitos mediais e laterais foi semelhante.

É possível observar que entre as enfermidades com maior ocorrência e que foram analisadas estatisticamente, houve diferença significativa quando comparados os sistemas de produção e as fêmeas primíparas e multíparas ( $\mathrm{p}<0,0001$ ) (Tabela 2). Dentre as doenças, observou-se que erosão de talão, sola plana, defeitos resultantes da laminite, erosão axial e sola dupla tiveram diferença significativa quando comparados os sistemas de produção ( $\mathrm{p}<0,0001)$. Na propriedade com sistema de produção de semiconfinamento houve maior índice de erosão de talão e erosão axial, diferindo dos resultados apresentados por Silva et al. (2001) que, ao trabalharem com bovinos das raças Holandesa, Jersey e Girolando em regime semiextensivo, encontraram como lesões mais frequentes a dermatite digital, seguida de dermatite verrucosa e pododermatite séptica. Tais lesões são de origem infecciosa, o que difere dos animais do presente estudo, nos quais as lesões são de origem mecânica. As lesões de erosão, tanto de talão como axial, a princípio são oriundas da abrasão do tecido córneo do casco, podendo ocasionar posteriormente infecção secundária. Já as lesões de dermatite verrucosa têm como principal agente infeccioso bactérias do gênero Treponema, e a pododermatite séptica é oriunda de um processo inflamatório, seja generalizado ou localizado, podendo ou não ser secundário a traumas (Borges et al., 2017). É de consenso geral que instalações em condições precárias de higiene podem atuar como fator predisponente das lesões podais infecciosas (Blowey, 1998). Existe, portanto, uma interface entre as questões infecciosas e o manejo das propriedades.

Já no sistema de produção free-stall houve um maior índice de defeitos resultantes da laminite, sola dupla e sola plana, diferentemente dos resultados obtidos por Nicoletti et al. (2001), que encontraram erosão axial e dermatite interdigital em maior ocorrência no sistemafree-stall. Resultados diferentes também foram obtidos por Tomasella et al. (2014), 
onde a lesão de maior ocorrência foi a úlcera de sola $(51,47 \%)$, seguida por doença da linha branca $(25,74 \%)$, sola fina $(10,29 \%)$, dermatite digital $(5,88 \%)$, dermatite interdigital $(4,41 \%)$, tiloma $(1,47 \%)$ e, por fim, hematoma de sola $(0,74 \%)$.

Em primíparas e multíparas, contudo, observouse diferença significativa $(\mathrm{p}<0,0001)$ somente entre a doença da linha branca e a sola plana, uma vez que nas primíparas do semiconfinamento houve diferença significativa. As primíparas do sistema de semiconfinamento apresentaram maior frequência de doença da linha branca em comparação com o outro sistema de criação e com as diferentes categorias animais (Albuquerque et al. 2009). Em multíparas do sistema free-stall é possível observar um aumento do índice de sola plana quando comparadas às fêmeas dos outros sistemas. No sistema de semiconfinamento, as lesões podais de maior prevalência nas vacas multíparas foram erosão de talão e erosão axial, com um índice de $40,48 \%$ e $36,19 \%$ respectivamente, superando os resultados encontrados por Pozzatti et al. (2010), relacionados ao encontro das lesões de erosão de talão, que obtiveram índice de 18,75\%.

Tabela 2 - Tipos de lesões das vacas primíparas e multíparas acometidas nos sistemas free-stall e semiconfinamento

\begin{tabular}{|c|c|c|c|c|c|c|c|c|c|c|c|c|}
\hline \multirow{3}{*}{ Tipos de lesões } & \multicolumn{6}{|c|}{ Free-stall } & \multicolumn{6}{|c|}{ Semiconfinamento } \\
\hline & \multicolumn{2}{|c|}{ Primíparas } & \multicolumn{2}{|c|}{ Multíparas } & \multicolumn{2}{|c|}{ Total } & \multicolumn{2}{|c|}{ Primíparas } & \multicolumn{2}{|c|}{ Multíparas } & \multicolumn{2}{|c|}{ Total } \\
\hline & FA & FR (\%) & FA & FR (\%) & FA & FR (\%) & $\mathrm{FA}$ & FR (\%) & FA & FR (\%) & FA & FR (\%) \\
\hline Hemorragia de sola & 1 & 0,46 & 1 & 0,46 & 2 & 0,5 & 5 & 1,66 & 4 & 0,95 & 9 & 1,25 \\
\hline Úlcera de sola & 0 & 0 & 0 & 0 & 0 & 0 & 0 & 0 & 1 & 0,24 & 1 & 0,14 \\
\hline Doença da linha branca & 14 & 6,39 a & 13 & 5,99 a & 27 & 6,19 & 9 & $2,99 \mathrm{~b}$ & 41 & $9,76^{a}$ & 50 & 6,93 \\
\hline Erosão de talão * & 65 & 29,68 & 63 & 29,03 & 128 & $29,36^{A}$ & 172 & 57,14 & 170 & 40,48 & 342 & $47,43^{\text {в }}$ \\
\hline Sola dupla * & 38 & 17,35 & 33 & 15,21 & 71 & $16,28^{A}$ & 0 & 0 & 5 & 1,19 & 5 & $0,69^{\mathrm{B}}$ \\
\hline Abscesso sub-solear & 0 & 0 & 0 & 0 & 0 & 0 & 0 & 0 & 0 & 0 & 0 & 0 \\
\hline Flegmão interdigital & 0 & 0 & 0 & 0 & 0 & 0 & 0 & 0 & 0 & 0 & 0 & 0 \\
\hline Tiloma & 0 & 0 & 0 & 0 & 0 & 0 & 0 & 0 & 1 & 0,24 & 1 & 0,14 \\
\hline Dermatite interdigital & 6 & 2,74 & 11 & 5,07 & 17 & 3,9 & 5 & 1,66 & 3 & 0,71 & 8 & 1,11 \\
\hline Dermatite digital & 2 & 0,91 & 3 & 1,38 & 5 & 1,15 & 4 & 1,33 & 2 & 0,48 & 6 & 0,83 \\
\hline Artrite (fístula/anquilose) & 0 & 0 & 0 & 0 & 0 & 0 & 0 & 0 & 0 & 0 & 0 & 0 \\
\hline Rachadura vertical & 4 & 1,83 & 0 & 0 & 4 & 0,92 & 0 & 0 & 4 & 0,95 & 4 & 0,55 \\
\hline Rachadura horizontal & 0 & 0 & 0 & 0 & 0 & 0 & 0 & 0 & 4 & 0,95 & 4 & 0,55 \\
\hline Pinça longa/Talão baixo & 4 & 1,83 & 6 & 2,76 & 10 & 2,29 & 0 & 0 & 3 & 0,71 & 3 & 0,42 \\
\hline Sola plana * & 27 & $12,33^{a}$ & 52 & $23,96^{b}$ & 79 & $18,12^{A}$ & 22 & $7,31^{\text {a }}$ & 20 & $4,76^{a}$ & 42 & $5,83^{\mathrm{B}}$ \\
\hline Defeitos resultantes da laminite * & 15 & 6,85 & 13 & 5,99 & 28 & $6,42^{\mathrm{A}}$ & 0 & 0 & 10 & 2,38 & 10 & $1,39^{\mathrm{B}}$ \\
\hline Erosão axial * & 43 & $19,63^{a}$ & 22 & $10,14^{b}$ & 65 & $14,91^{\mathrm{A}}$ & 84 & $27,91^{b}$ & 152 & 36,19 a & 236 & $32,73^{B}$ \\
\hline
\end{tabular}

Nota: * Enfermidades com maior ocorrência e que foram analisadas estatisticamente. Frequências na mesma linha com letras diferentes diferem entre si $(p<0,05)$. FA = frequência absoluta; $F R=$ frequência relativa.

Nas vacas multíparas mantidas no sistema freestall houve maior prevalência para as lesões de erosão de talão $(29,03 \%)$, sola plana $(23,96 \%)$ e sola dupla $(15,21 \%)$. 0 índice de lesões de sola dupla $(4,3 \%)$ encontrado por Pozzatti et al. (2010) difere inferiormente dos encontrados no presente estudo.
Em relação às demais doenças, não foi possível calcular as possíveis diferenças estatisticamente relevantes devido à baixa incidência das mesmas, assim como foi possível observar a ausência de abcesso sub-solear, flegmão interdigital e artrite nos animais do presente estudo. Contudo, Silva 
et al. (2013) encontraram a dermatite digital promovendo alteração importante na relação comportamental de fêmeas criadas em regime extensivo, com diminuição no tempo de pastejo e ruminação.

De acordo com Silva et al. (2004), as mudanças na alimentação, a não utilização de tamponantes na dieta, a alta concentração de animais por área, favorecendo o acúmulo de matéria orgânica e umidade, a falta ou o uso incorreto do pedilúvio e a falta de casqueamento preventivo foram considerados os maiores fatores de risco para a introdução de enfermidades podais. Em ambas as propriedades onde se desenvolveu este estudo, ocorria rotineiramente a mudança brusca na alimentação, trocando um componente da dieta por outro conforme a disponibilidade, sem adaptação prévia. Soma-se a isto o fato de que não usar tamponantes na dieta pode predispor a problemas metabólicos, que podem levar à laminite subclínica. 0 uso de tamponantes tem como objetivo diminuir as variações de $\mathrm{pH}$ do rúmen, causado pela ingestão de alimentos concentrados ricos em carboidratos não estruturais, mantendo os parâmetros nas condições normais em função da fermentação ruminal (Ortolan, 2010).

\section{Conclusão}

Conclui-se que o sistema de produção das duas propriedades utilizadas neste estudo não diferiram entre si na ocorrência de afecções podais em vacas lactantes da raça Jersey. Além disto, a existência de lesões podais não foi correlacionada diretamente à claudicação.

\section{Referências}

Albuquerque PI, Ximenes FHB, Moscardini ACR, Gouvêa LV, Mota ALAA, Godoy RF, et al. Caracterização das afecções podais em rebanho de gado holandês confinado. Cienc Anim Bras. 2009;7(Supl 1):46-52.

Alves CGT, Silva FF, Melo LEH, Santos NVM, Silva Jr FF, Machado PP. Susceptibilidade de fêmeas leiteiras às afecções podais. Med Vet (UFRPE). 2007;1(1):14-8.
Blowey RW. Welfare aspects of foot lameness in cattle. Compendium on Continuing Education for the Practicing Veterinarian. Ir Vet J. 1998;51(4)203-7.

Borges JRJ, Garcia M. Guia Bayer de podologia bovina. São Paulo: Bayer; 2002. 45 p.

Borges JRJ, Câmara ACL, Moscardini ARC, Rodrigues CA, Pitombo CA, Graça FA, et al. Doenças dos dígitos dos bovinos: nomenclatura padronizada para o Brasil. CFMV. 2017;23(73):45-52.

Borkert J. Lameness in pasture $\mathrm{v} / \mathrm{s}$ confined system: what the Chilean experience says? 18th International Symposium \& 10th Conference onLameness in Ruminants;22-25 nov 2015; Valdivia, Chile. Valdivia: Universidad Austral de Chile; 2015. p. 63.

Cerqueira JL, Araújo JP, Sorensen JT, Niza-Ribeiro J. Alguns indicadores de avaliação de bem-estar em vacas leiteiras - revisão. RPCV. 2011;106(577-590):5-19.

Costa DNM, Santos HP, Bezerra AS, Silva CRA, Barbosa RD, Feitosa FS, et al. Aspectos clínicos do flegmão intersticial bovino. PUBVET. 2014; 8(10):1136-282.

Greenough P. Bovine laminitis and lameness: A hands-on approach. Philadelphia: Saunders; 2007. 319 p.

Greenough PR, Weaver AD. Lameness in Cattle. Philadelphia: Saunders; 1997. 336 p.

Martins MF, Pieruzzi PAP. Bem estar animal na bovinocultura leiteira. In: Santos MV et al. (EE.). Novos desafios da pesquisa em nutrição e produção animal. Pirassununga: 5D Editora; 2011. p. 212-28.

Nicoletti JLM. Manual de podologia bovina. Barueri: Manole; 2004. 130 p.

Nicoletti JLM, Souza FAA, Thomassian A, Hussni CA, Alves ALG. Prevalência de lesões podais e graus de claudicação em vacas leiteiras mantidas em confinamento permanente ("'free-stall" e "'tie-stall'). MV\&Z. 2001;4(2):24-32.

Ortolan JH. Efeito de aditivos no metabolismo ruminal e parâmetros sanguíneos em bovinos [tese]. Pirassununga: Universidade de São Paulo; 2010.67 p. 
Plautz GR. Podologia bovina [monografia]. Porto Alegre: Universidade Federal do Rio Grande do Sul; 2013. 59 p.

Pozzatti PN, Casagrande FP, Porfírio LC, Amorim JB, Ferreira LO, Lago LA, et al. Prevalência e classificação das afecções podais de rebanho bovino leiteiro de Sete Lagoas - MG. XIII Encontro Latino Americano de Iniciação Científica e IX Encontro Latino Americano de Pós-Graduação. 21-22 out 2010; São José dos Campos, São Paulo. São José dos Campos: Universidade o Vale do Paraíba; 2010. p. 123.

Roman RC, Nunes MLA, Oltramari CE, Zotti CA. Prevalência de problemas de casco em vacas leiteiras na região de Chapecó/SC encontrados em sistemas de confinamento e semiconfinamento. Enc Biosfera. 2014;10(19):1533.

Romani AF, Silva LAF, Fioravanti MCS, Rabelo RE, Cunha PHJ, Amaral AVC, et al. Ocorrência de lesões podais em fêmeas bovinas leiteiras no estado de Goiás. Ars Vet. 2004;20(3):322-9.

Rutter B. Patologías podales infecciosas y no infecciosas em vacas lecheras. Maskana. 2015;6(Supl): 119-29.

Silva LAF, Coelho KO, Machado PF, Silva MAM, Moura MI, Barbosa VT, et al. Causas de descarte de vacas da raça Holandesa confinadas em uma população de 2.083 bovinos (2000-2003). Cienc Anim Bras. 2008;9(2):383-9.
Silva LAF, Cunha PHJ, Noronha Filho ADF, Goulart DS, Campos SBS, Freitas SLR. Comportamento diário de vacas da raça Girolando com dermatite digital manejadas extensivamente. Vet Zootec. 2013;20(3):459-66.

Silva LAF, Fioravanti MCS, Trindade BR, Silva OC, Eurides D, Cunha PHJ, et al. Enfermidades digitais em vacas de aptidão leiteira: associação com mastite clínica, metrites e aspectos epidemiológicos. Pesq Vet Bras. 2004;24(4):217-22.

Silva AAM, Oliveira Filho JP, Pimenta-Oliveira A, Badial PR, Cunha PHJ, Souza FAA. Prevalência e distribuição clínica de lesões digitais em vacas da raça Holandesa mantidas em sistema "free-stall". PUBVET. 2010;4(7):752-8.

Silva LAF, Silva LM, Romani AF, Rabelo RE, Fioravanti MCS, Souza TM, et al. Características clínicas e epidemiológicas das enfermidades podais em vacas lactantes do município de Orizona - GO. Cienc Anim Bras. 2001;2(2):119-26.

Souza AM, Tulio LM, Gai VF. Incidência de lesões podais em bovinos de aptidão leiteira na região oeste do Paraná. Rev Cult Saber. 2015;1:194-202.

Tomasella TE, Negri Filho LC, Affonso MZ, Barca Jr F, Silva LC, Okano W. Prevalência e classificações de lesões podais em bovinos leiteiros na região de Belo Horizonte - MG. Rev Bras Hig San Anim. 2014;8(1):115-27. 